\title{
SUPERCONDUCTING FINAL FOCUSING SYSTEM FOR KEKB
}

\author{
K. Tsuchiya, N. Ohuchi, T. Ogitsu, T. Ozaki, R. Sugahara, KEK, Tsukuba, Ibaraki, Japan
}

\begin{abstract}
A superconducting final focusing system for the KEKB B-Factory was constructed and has been operating since 1998. It consists of two solenoid field compensation magnets, two final focusing quadrupole magnets, and three kinds of correction coils (normal and skew dipoles, and skew quadrupole). The system has been operating without any serious problems and its total operating time has reached 17,000 hours. This paper describes the details of the system and operating experiences gathered during the long-term operation.
\end{abstract}

\section{INTRODUCTION}

KEKB is an asymmetric, two-ring electron-positron collider, which circulates $8 \mathrm{GeV}$ electron and $3.5 \mathrm{GeV}$ positron beams in opposite directions, and collides them at one interaction point (IP) with a finite crossing angle of $\pm 11 \mathrm{mrad}$. A schematic layout of the beam line near the IP is shown in Fig. 1. This figure shows the four superconducting magnets (S-R, S-L, QCS-R, QCS-L) and two normal-conducting quadrupole magnets (QC1RE and QC1LE). The final vertical focusing of two beams is provided by a pair of superconducting quadrupoles (QCS$\mathrm{R}$ and $\mathrm{QCS}-\mathrm{L}$ ). The solenoids (S-R and S-L) are for compensating a solenoid field created by the Belle detector. The QCS and S magnets on each side of the IP are contained in a common cryostat. The vertical focusing strength of QCS is tuned to the positron beam. Extra vertical focusing for the electron beam is provided by a pair of quadrupoles (QC1RE and QC1LE). The geometries of these quadrupoles were designed such that only the electron beam would see the quadrupole fields when passing through them.

Positrons enter the IP through QCS-R and leave through QCS-L. Electrons proceed in the opposite direction. To minimize the synchrotron radiation background near the IP, the magnetic axes of S-R and QCS-R were aligned on the same line, and the QCS-L axis was

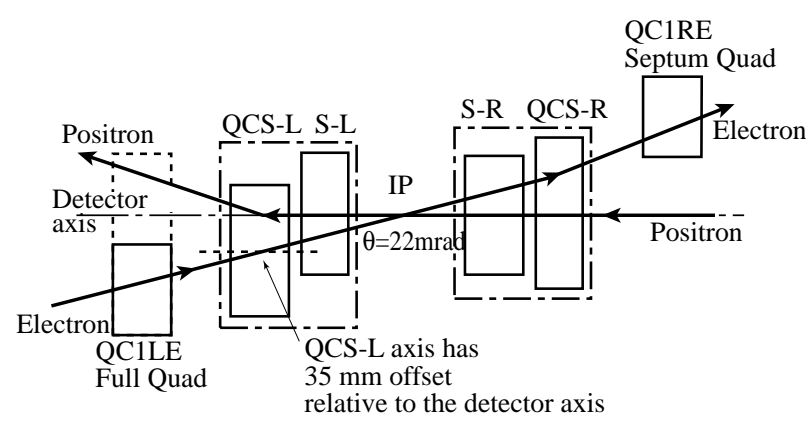

Figure 1: Schematic layout of the beam line near IP.

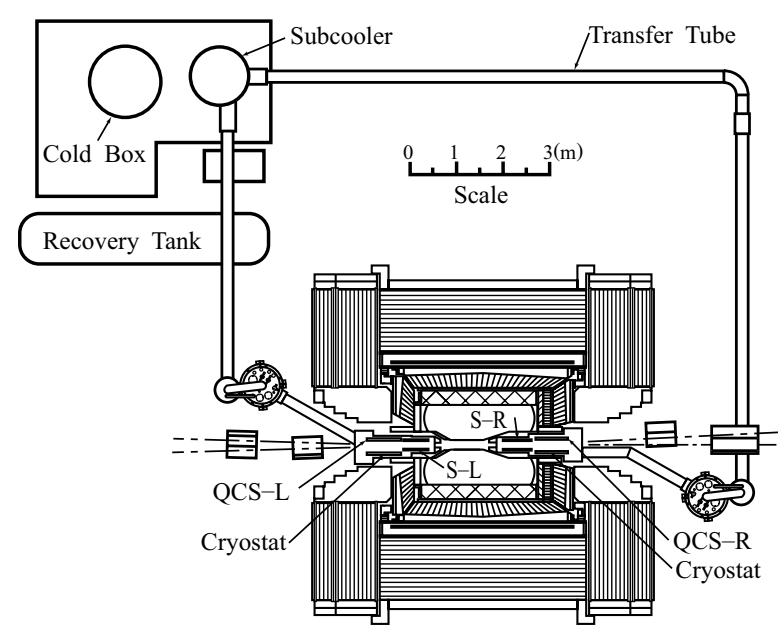

Figure 2: Layout of the superconducting final focusing system for KEKB, together with the Belle detector facility.

shifted by $35 \mathrm{~mm}$ with respect to the axis of the S-L [1].

Figure 2 shows the layout of the superconducting final focusing system. As can be seen in this figure, the locations of the cryostats containing QCS and S magnets are completely inside the detector. Therefore, they must be compact and rigidly supported to withstand large electromagnetic forces applied on the coils from the $1.5 \mathrm{~T}$ detector solenoid. The magnet cryostats are held by rigid cantilever supports.

A service duct, which comes out from the cryostat, is connected to a service cryostat which is located outside the detector. Another multi-channel transfer line runs down from here and extends horizontally towards a subcooler-cold box unit, which sits at the side of the Belle detector.

\section{COMPENSATION MAGNET}

Two compensation solenoids (S-R and S-L) were installed at the closest locations to the IP. Their magnetic field direction is opposite to the detector solenoid, and minimizes the effect of the detector solenoid field on the accelerator beams. Table 1 summarizes the main parameters of the magnets. The design parameters of these solenoids were selected to satisfy a condition that an integral of $\mathrm{Bz}$ along the beam line becomes zero.

Because of the presence of the detector solenoidal field, the peak fields on the conductors and the central fields in normal operation are reduced by $1.5 \mathrm{~T}$ from the values listed in Table 1, and the axial repulsive forces work on the solenoid. The calculated forces on S-L and S-R are 22 $\mathrm{kN}$ and $2.8 \mathrm{kN}$, respectively. Since these solenoids are 
Table 1: Main Parameters of Compensation Magnets.

\begin{tabular}{|l|l|l|}
\hline & S-L & S-R \\
\hline Central field (T) & 4.53 & 5.80 \\
\hline Current (A) & 487 & 603 \\
\hline I/Ic @ 4.5 K (\%) & 59 & 75 \\
\hline Bmax on the conductor (T) & 4.59 & 5.83 \\
\hline Stored energy (kJ) & 121 & 258 \\
\hline Coil length (mm) & 461 & 616 \\
\hline inner dia. (mm) & 190 & 190 \\
\hline outer dia. (mm) & 230 & 230 \\
\hline No. of turns & 3749 & 4981 \\
\hline
\end{tabular}

excited in a field of $1.5 \mathrm{~T}$, the current dependence of the radial bursting forces on the coil is quite different compared with that of ordinary solenoids [2]. A rather thick bobbin (helium inner cylinder) of $7 \mathrm{~mm}$ thickness was selected and the windings were impregnated with epoxy resin in order to manage such forces.

The coils were made of monolithic NbTi superconducting wire with a cross section of $1.1 \mathrm{~mm} \times 1.9 \mathrm{~mm}$ $(\mathrm{Cu} / \mathrm{SC}$ ratio 1.0$)$. The insulation is provided by polyimide group resin. The coils were impregnated with epoxy resin under pressure after being wound on a helium inner cylinder, and enclosed in helium vessels while axial compression forces were applied. The expected axial compression forces on the assembled coils were $516 \mathrm{MN}$ and $250 \mathrm{MN}$ for S-R and S-L, respectively.

During the first test in a vertical cryostat, the S-R magnet required a number of training quenches to reach the design current. However, the S-L magnet reached the design current without quenching. After installation into the horizontal cryostat, both magnets showed good performance; no training quenches were required to reach their design currents with and without the $1.5 \mathrm{~T}$ detector solenoid field.

The $\mathrm{Bz}$ field profile along the positron beam line was measured with a Hall probe. The results are shown in Fig. 3 when the S-L, S-R and the detector solenoid were all excited.

\section{QUADRUPOLE MAGNET WITH CORRECTION COILS}

The quadrupole magnets are iron-free superconducting magnets [2],[3]. They produce a field gradient of 21.7 $\mathrm{T} / \mathrm{m}$ within a coil aperture diameter of $260 \mathrm{~mm}$. The coil

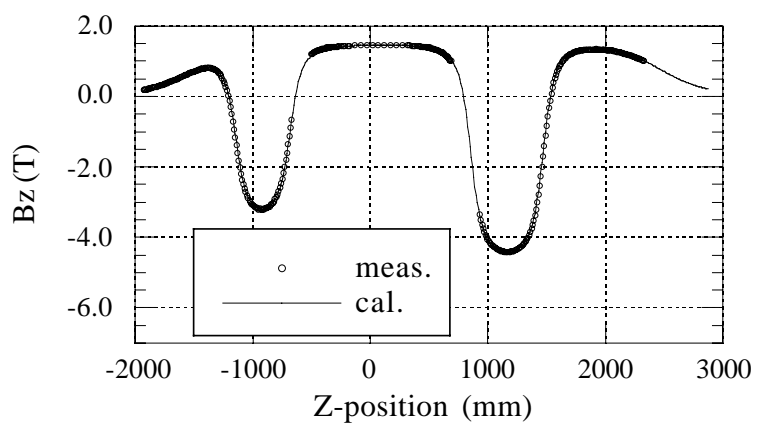

Figure 3: Bz distribution along the beam line.
Table 2: Main Parameters of Quadrupole Magnets.

\begin{tabular}{|l|l|l|}
\hline & QCS-L & QCS-R \\
\hline Field gradient (T/m) & $21.66(21.84)$ & $21.73(21.84)$ \\
\hline Current $\quad(\mathrm{A})$ & 2963 & 2963 \\
\hline Effective length $(\mathrm{mm})$ & $483(486.1)$ & $385(387.7)$ \\
\hline I/Ic @ 4.5K (\%) & 70 & 70 \\
\hline Bmax on the cable $(\mathrm{T})$ & 4.3 & 4.3 \\
\hline Stored energy (kJ) & 87.5 & 69.7 \\
\hline Coil length (mm) & 617 & 521 \\
\hline inner dia. (mm) & 260 & 260 \\
\hline outer dia. (mm) & 289.8 & 289.8 \\
\hline Collar outer dia. $(\mathrm{mm})$ & 340 & 340 \\
\hline
\end{tabular}

Numbers within parentheses are measured values.

design is based on a set of $\cos 2 \theta$ windings that are clamped by stainless-steel collars. The two-dimensional structure for both QCS-L and QCS-R are the same, while their lengths are slightly different. Since these quadrupoles are rather short and have a large aperture, we performed three-dimensional field calculations in order to optimize the shape of the coil end. Table 2 lists the main parameters of the quadrupoles and Fig. 4 shows the transverse cross section of the QCS-L magnet. The inner diameter of the warm bore is $160 \mathrm{~mm}$ and the outer diameter of the cryostat is $480 \mathrm{~mm}$. From the innermost part, the main components are: a warm bore, inner thermal radiation shield, inner wall of the helium vessel, correction coils, main quadrupole coil, $316 \mathrm{LN}$ stainlesssteel collar, outer wall of the helium vessel, outer thermal radiation shield and vacuum vessel. The small holes on the collar are for the bus lines and the instrumentation wires of the $\mathrm{S}$ magnet.

We used a $\mathrm{NbTi} / \mathrm{Cu}$ Rutherford cable for these magnets. It consists of 24 multifilamentary strands of $0.59 \mathrm{~mm}$ diameter $(\mathrm{Cu} / \mathrm{SC}$ ratio 1.8$)$ twisted with a pitch of about $60 \mathrm{~mm}$ (filament size $6 \mu \mathrm{m}$ ). The residual resistivity ratio of the stabilizing copper is about 160 . The width and the mid-thickness of the bare cable are $7.000 \pm 0.025 \mathrm{~mm}$ and $1.090 \pm 0.006 \mathrm{~mm}$, respectively. The cable is insulated with two kinds of Upilex tape: $25 \mu \mathrm{m}$ tape and $50 \mu \mathrm{m}$ tape with B-stage epoxy resin.

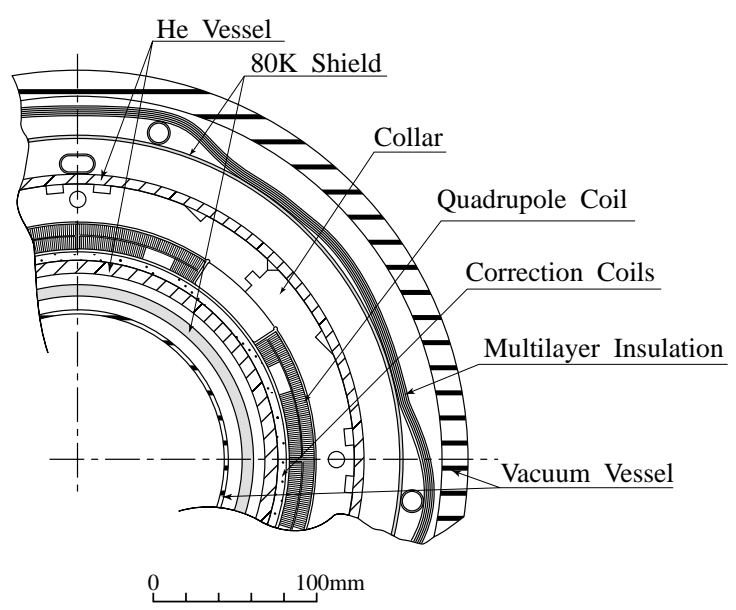

Figure 4: Cross section of the quadrupole magnet. 
The final field measurements of QCS magnets were performed after they were installed into the beam line. We measured the field distribution along the magnetic axis and the integral field using two kinds of harmonic coils, one with $50 \mathrm{~mm}$ long and other with $800 \mathrm{~mm}$ long. The radii of the harmonic coils are $40 \mathrm{~mm}$. The multipole field of the QCS-R was measured by setting the harmonic coil axis on the magnet center. However, that of the QCS-L was measured around the axis of the S-L magnet that is shifted by $35 \mathrm{~mm}$ from the QCS-L field center. Figure 5 shows the integral multipole coefficients of these magnets [4].

Three correction coils (normal and skew dipole coils, and skew quadrupole coil) are embedded inside the quadrupole winding. The field strength of the dipole is about $0.05 \mathrm{~T}$ at $50 \mathrm{~A}$ and the gradient of the skew quadrupole is about $0.4 \mathrm{~T} / \mathrm{m}$ at $50 \mathrm{~A}$. The effective lengths are about 440 and $500 \mathrm{~mm}$ for the dipoles and the skew quadrupole, respectively. With these strengths, we can rotate the quadrupole field by $\pm 10 \mathrm{mrad}$ and shift its center by $\pm 3 \mathrm{~mm}$.

The fabrication method was similar to that of the corrector magnets for RHIC [5]. First, one-layer coils were wound on an epoxy-coated flat substrate by using a multi-wiring technique. These flat coils were made at BNL using a $0.33 \mathrm{~mm}$ diameter superconducting wire covered with $25 \mu \mathrm{m}$ thick Kapton insulation. Then, the coils were assembled onto the helium inner cylinder by a Japanese company. The total thickness for three correction coils, including the insulation and the bindings of the coils, was $6.6 \mathrm{~mm}$. Since these coils are
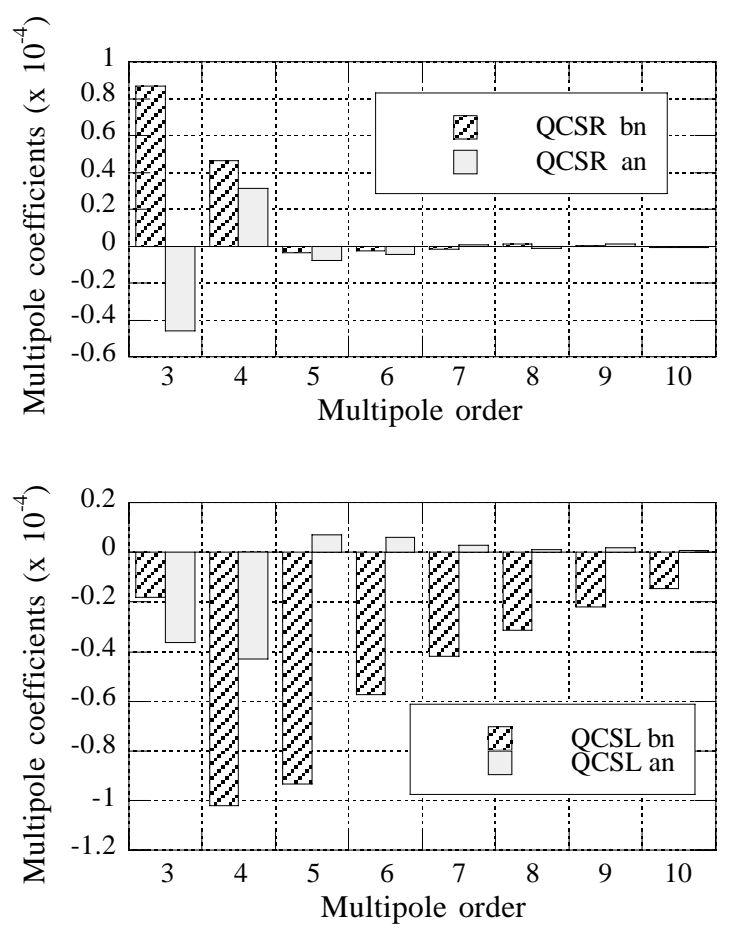

Figure 5: Integral multipole coefficients of QCS magnets at $3000 \mathrm{~A}$. placed inside the quadrupole and solenoid, various forces and torques are exerted on each coil [2]. We used a relatively thick stainless-steel cylinder $(8 \mathrm{~mm})$ for the helium inner vessel in order to manage these forces.

\section{CRYOSTAT}

Severe spatial constraints were imposed on the cryostat design and very compact cryostats had to be built [6], because they are placed almost inside the detector. In addition, in the case of the left-side cryostat, the quadrupole axis had to be shifted horizontally by $35 \mathrm{~mm}$ from the solenoid axis. This introduced significant difficulty in the fabrication and assembly. Figure 6 shows a cross sectional view of the left-side cryostat.

The cryostat system consists of three parts: a horizontal annular vessel (magnet cryostat) containing the magnets, a service cryostat, and a service duct connecting both cryostats. The helium vessel is made of $316 \mathrm{~L}$ stainless steel. The inner cylinder works not only as a part of the pressure vessel, but also as a mechanical support of the coils. The helium vessel is surrounded by a thermalradiation shield. The outer shield consists of a stainlesssteel shell with cooling pipe, while the inner shield consists of a $7 \mathrm{~mm}$ thick 316L stainless-steel cylinder with helical grooves for liquid nitrogen. About 30 layers of aluminized Kapton were arranged between the vacuum vessel and the thermal shield. However, since the spatial separation between the helium vessel and the thermal shield is limited (6-7 mm gap), no superinsulation was installed.

The helium vessel containing the solenoid and the quadrupole was suspended in the vacuum vessel by eight support rods. The rods were designed to withstand large magnetic forces which arise from various Lorentz forces due to combined operation of the magnets. They are made of titanium alloy, Ti-6Al-4V [ELI], which has a high ultimate tensile strength and a low thermal conductivity.

The service cryostat functions as an interface between the cryostat and the multi-channel transfer line coming from the subcooler box. On the top flange of the service cryostat there are bayonet joints for transfer lines, current leads for solenoid and correction coils, two control valves, and some service ports. The current leads for QCS magnets are not on the service cryostat. They are on the subcooler box, and the current is supplied to the QCS magnets via superconducting bus lines in the multichannel transfer line.

During a performance test, the heat load of each cryostat system was evaluated from the mass flow rate and the temperature increase in the cryostat. The values were 12.1 and $10.2 \mathrm{~W}$ for the right and left side cryostat. These are slightly less than the design values.

\section{ELECTRICAL SYSTEM}

A schematic diagram for the electrical circuit is shown in Fig. 7. The SCR power supply for the QCS magnets is composed of a main power unit $(3500 \mathrm{~A}, 15 \mathrm{~V})$ and an 

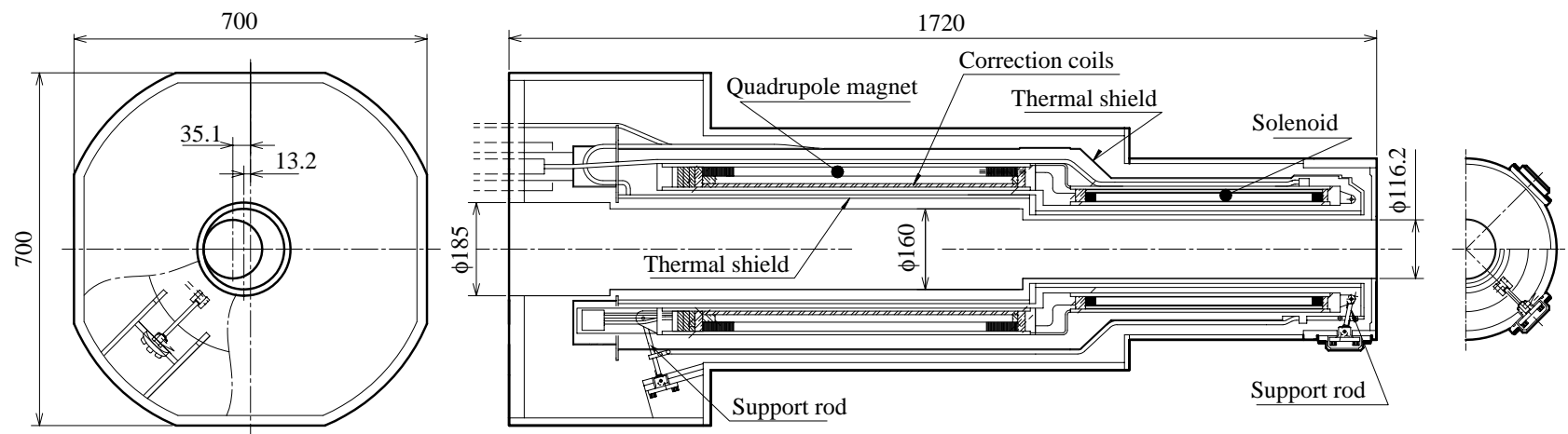

Figure 6: Cross section of the L-side cryostat.

auxiliary power unit (40A, 15V). Two QCS magnets are excited in series by this power supply, while other magnets have their own power supplies.

The quench protection circuit of QCS is composed of a high-speed SCR switch and two energy dump resistors. The values of the resistors for QCS-R and QCS-L were set at $0.1 \Omega$ and $0.12 \Omega$, respectively, in order to optimize the time constants of the current decay of QCS-R and QCS-L. For quench protection of other magnets, we used DC circuit breakers since they did not require rapid decay of the currents in case of quenching. The selected resistors were $0.71 \Omega$ and $2 \Omega$ for the solenoid and the correction coil, respectively. The typical quench threshold is set at $1 \mathrm{~V}$ for $10 \mathrm{~ms}$ or more.

\section{COOLING SYSTEM}

The main components of the cooling system are of the TRISTAN mini-beta insertion quadrupole magnet system [7], although the cryostats and some parts of the transfer lines were renewed. The cooling capacity of this system is $150 \mathrm{~W}+30 \mathrm{~L} / \mathrm{h}$. The magnets are cooled by a singlephase LHe of $4.5 \mathrm{~K}$ at a flow rate of $21 \mathrm{~g} / \mathrm{sec}$ during steady state operation.

Cool-down is achieved by forced circulation of cold gaseous helium. The gas temperature is gradually lowered in order to follow the magnet temperature. Cool-down takes about 27 hours, during which time the peak

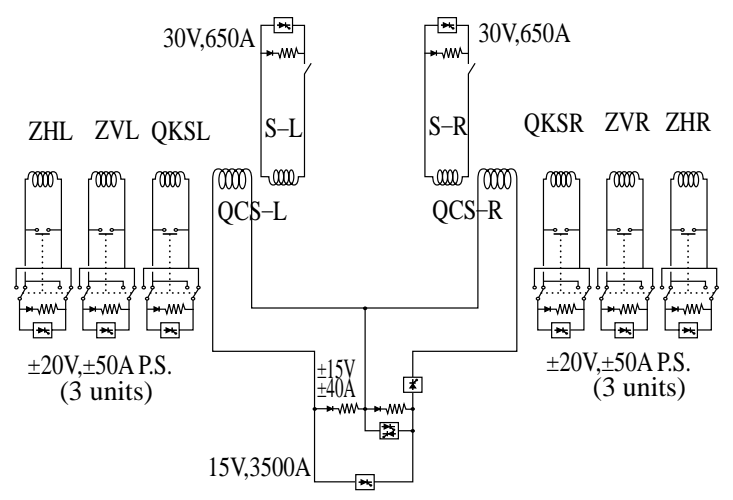

Figure 7: Electric circuit of the magnet system. temperature difference between the magnets and gas is $50 \mathrm{~K}$.

Figure 8 shows a typical cool-down curve of the magnet system. The cooling system performed well even during and after quenching at full current excitations. The heat loads of the system evaluated from the redundant cooling power of the refrigerator was about $75 \mathrm{~W}+30 \mathrm{~L} / \mathrm{h}$.

\section{OPERATIONAL EXPERIENCE}

Five superconducting coils in each cryostat and presence of the $1.5 \mathrm{~T}$ external field generate large net Lorentz forces and torques on the magnet as a whole. We measured the movement of each cryostat during excitation. A large effect can be seen when the S magnet or the dipole correction coil is excited when the detector solenoid is on. The front ends of the L-side and R-side cryostat moved upward by $0.58 \mathrm{~mm}$ and downward by $0.1 \mathrm{~mm}$, respectively, at their operating currents. When the dipole correction coils are excited, the cryostat moved by 0.2 to $0.3 \mathrm{~mm}$ either vertically or horizontally depending on the kinds of dipole coil (normal or skew). However, these movements are not so serious, since they are stable and reproducible effects.

The cooling system is controlled by a computerized and automated system. Since the KEKB was commissioned, the system was cooled down eight times. Figure 9 shows

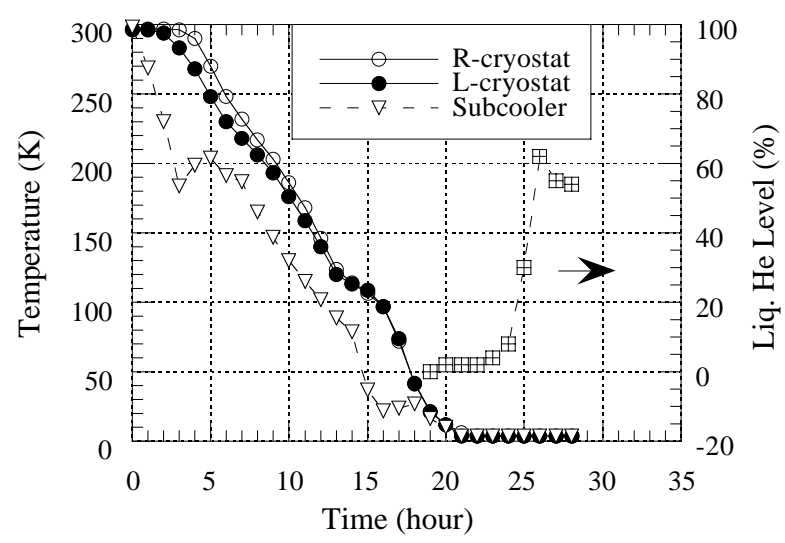

Figure 8: Cool-down curve of the system. 


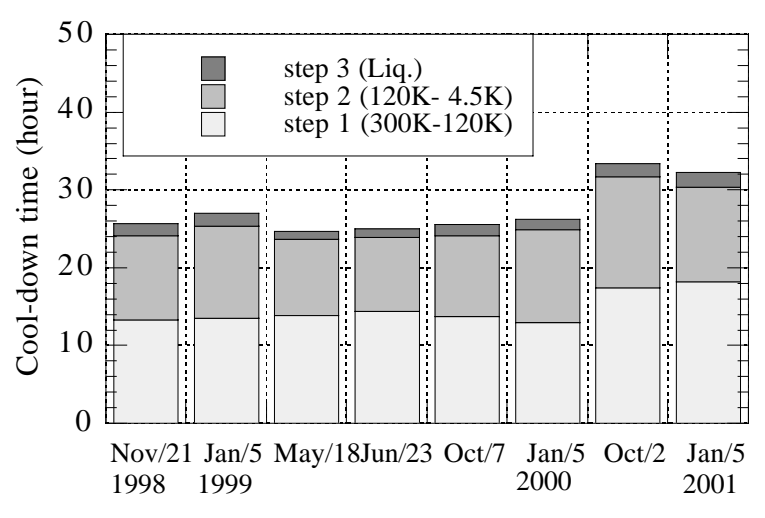

Figure 9: Cool-down time of the system.

the elapsed time of each cool-down process. A relatively stable cool-down can be seen in the figure, although in the last two cool-down slightly longer times were required. This was caused by an incorrectly opened valve in the LN2 supply line in the initial step of cool-down.

When the quench protection circuit is triggered due to a magnet quench or power supply trouble, the cooling of the cryostats stops automatically and the cryostats are isolated from the cooling system. After settling the temperature rise of the magnet, the cryostat is re-cooled and recovered to the normal operating condition. The average recovery time is 3.5 hours.

Another concern in a long-term operation of the superconducting magnet system is failures of the components. In Fig. 10 a summary of failures that occurred during past three years is shown. We classified them in five categories: cryogenic plant (compressors, cryogenic equipments which include valves and monitors), utility (cooling water system, air compressor), other sources (accelerator safety interlock, mis-operation, etc.), electric power system (by thunderstorm, earthquake, etc.), and quench (beam induced quench, quench detection system and power supply trouble). Although the total number of failures is not so small, in many cases the continuous operation of the system was not interrupted. The number of those that interrupted normal operation and required an operator's assistance to return to normal is listed in the parentheses. Most of these problems in 1999 and 2000 were due to thunderstorm, earthquake, and power supply problems. Therefore, we improved the power supplies to be less sensitive to the $\mathrm{AC}$ line voltage change in October 2000.

\section{SUMMARY}

A superconducting final focusing system for KEKB was constructed and has been operated during past three years. Its total operating time has reached about 17,000 hours.

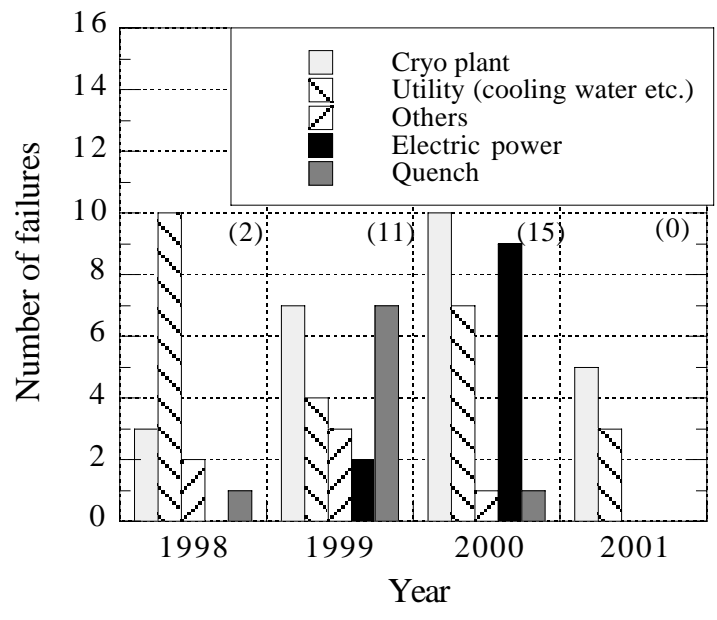

Figure 10: Summary of failures.

Number of failures that interrupt normal operation of the system is listed in parentheses.

In spite of its complexity, this system of ten superconducting magnets and cooling system has been working very stably without experiencing any serious problems. By improving the interlock system of the power supplies, we would like to reduce the downtime of the system even more.

\section{REFERENCES}

[1] KEKB B-Factory Design Report, KEK Report 95-7 (1995).

[2] K. Tsuchiya et al., "Superconducting magnet System for KEKB B-Factory," Proc. of the 5th European Particle Accelerator Conference (EPAC'96), p. 2287.

[3] K. Tsuchiya et al., "Superconducting magnets for the Interaction Region of KEKB," IEEE Trans. Appl. Superconductivity, Vol. 9, No. 2 (1999), p.1045.

[4] N. Ohuchi et al., "The performance of the superconducting magnet system in the KEKB interaction region," Proc. of e+e- Factories'99, p.217, KEK Pro. 99-24.

[5] A. Morgillo et al., "Superconducting $8 \mathrm{~cm}$ Corrector Magnets for the Relativistic Heavy Ion Collider (RHIC),' Proc. of the 1995 PAC and Int. Conf. on High-Energy Accelerators, p.1393.

[6] N. Ohuchi et al., "Cryostats for the KEKB IR Superconducting Magnets," Adv. in Cryo. Eng., Vol. 45 (2000), p.787.

[7] K. Tsuchiya et al., "Helium Cryogenic Systems for the Superconducting Insertion Quadrupole Magnets of the TRISTAN Storage Ring," Adv. in Cryo. Eng., Vol. 37A (1992), p.667. 